\title{
The effect of gene transfer with hepatocyte growth factor for pulmonary vascular hypoplasia in neonatal porcine model
}

\author{
Masamichi Ono, MD, PhDa \\ Yoshiki Sawa, MD, PhD \\ Yuji Miyamoto, MD, $\mathrm{PhD}^{\mathrm{a}}$ \\ Norihide Fukushima, MD, $\mathrm{PhD}^{\mathrm{a}}$ \\ Hajime Ichikawa, MD ${ }^{\mathrm{a}}$ \\ Toru Ishizaka, MD, PhD \\ Yasufumi Kaneda, MD, $\mathrm{PhD}^{\mathrm{b}}$ \\ Hikaru Matsuda, MD, $\mathrm{PhD}^{\mathrm{a}}$
}

From the Division of Cardiovascular Surgery, Departments of Surgery a and Gene Therapy Science, ${ }^{\mathrm{b}}$ Osaka University Graduate School of Medicine, Osaka, Japan.

This work was supported by a Grant-in-Aid for Scientific Research in Japan and Grant from Japan Cardiovascular Research Foundation (the 10th Bayer scholarship for cardiovascular research).

Received for publication Feb 17, 2004; revisions received May 15, 2004; accepted for publication June 17, 2004.

Address for reprints: Yoshiki Sawa, Division of Cardiovascular Surgery, Department of Surgery, Osaka University Graduate School of Medicine, 2-2 Yamadaoka, Suita, Osaka 565-0871, Japan (E-mail: sawa@surg1.med.osaka-u.ac.jp).

J Thorac Cardiovasc Surg 2005;129:740-5 $0022-5223 / \$ 30.00$

Copyright $\odot 2005$ by The American Association for Thoracic Surgery

doi:10.1016/j.jtcvs.2004.06.040
Background: Severe degree of pulmonary vascular hypoplasia remains a major limitation in congenital heart surgery. Considering the potential effect of gene transfer with hepatocyte growth factor to induce the angiogenesis in the lung, we assessed the effects of hepatocyte growth factor gene transfer in neonatal porcine lung with pulmonary vascular hypoplasia to achieve treatment for severe pulmonary vascular hypoplasia.

Methods: The model of pulmonary vascular hypoplasia was introduced with left pulmonary artery banding in piglet lung. After 7 days of pulmonary artery banding, piglets were transfected selectively to the left lung via the left pulmonary artery with a hemagglutinating virus of Japan E vector bearing the cDNA encoding human hepatocyte growth factor ( $\mathrm{H}$ group) or control vector (C group).

Results: Seven days after the transfection, selective angiography of the left pulmonary artery showed the progression of left pulmonary vascular hypoplasia of the left lung in the $\mathrm{C}$ group but a significant attenuation of left pulmonary vascular hypoplasia in the $\mathrm{H}$ group. A right pulmonary artery occlusion test showed a marked increase in right ventricular systolic pressure in the $\mathrm{C}$ group, but this was significantly attenuated in the $\mathrm{H}$ group $(\mathrm{C}: 22.0 \pm 2.9, \mathrm{H}: 13.0 \pm 2.7 \mathrm{~mm} \mathrm{Hg} ; P<.05)$. Histologic examination revealed that hepatocyte growth factor gene transfection increased the pulmonary vasculature in the left lung.

Conclusions: Our results demonstrated that gene transfer of hepatocyte growth factor via the pulmonary artery showed the angiogenic effects in porcine model of pulmonary vascular hypoplasia after pulmonary artery banding.

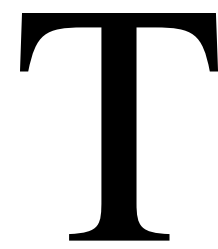

he feasibility of a definitive surgical repair of cyanotic congenital heart disease largely depends on the development of the pulmonary vasculature. Since the introduction of the Blalock-Taussig shunt, ${ }^{1}$ the strategy for treatment of hypoplastic pulmonary artery has been established, and the surgical advances that followed improved the clinical outcomes of patients with hypoplastic pulmonary artery. ${ }^{2-5}$ However, a group of patients still remains who survive palliation but cannot undergo definitive surgery and who require further treatment for progressive cyanosis. ${ }^{6}$ These patients are usually associated with hypoplasia within the entire pulmonary vascular bed as well as hypoplasia of central pulmonary arteries. In this context, 

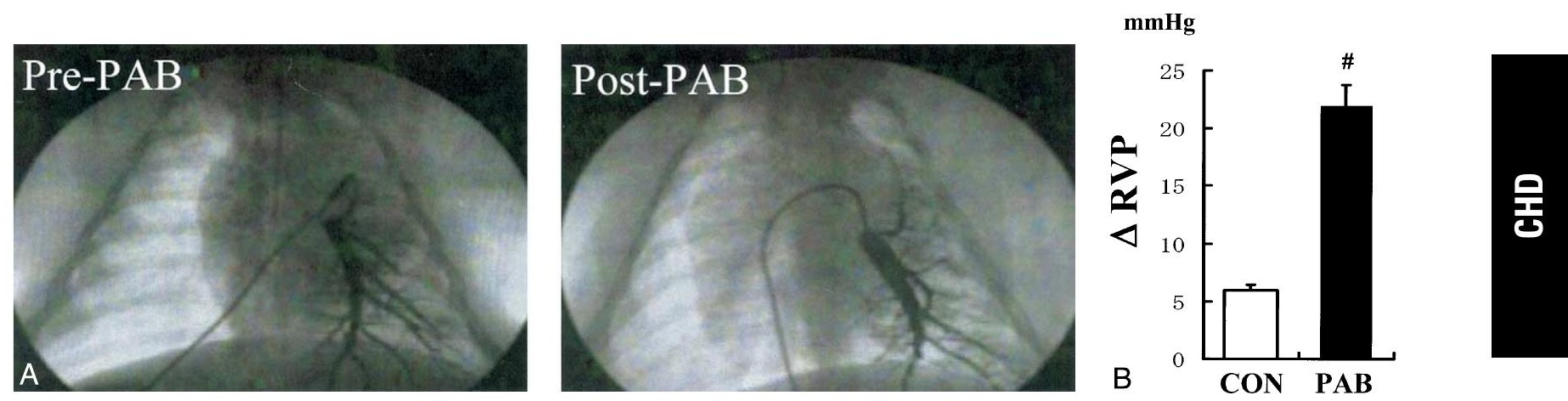

Figure 1. Evaluation of pulmonary vascular hypoplasia by left pulmonary artery banding (PAB). A, Pulmonary angiogram. Selective left pulmonary arteriogram 7 days after left pulmonary artery banding shows pulmonary vascular hypoplasia (right) compared with the normal control (left). B, Right pulmonary artery occlusion test. Changes in right ventricular systolic pressure $(\Delta R V P)$ reveal a significant increase after pulmonary artery banding. Each value represents the mean \pm SEM $(n=4)$. \#P<.05 versus control (Student $t$ test). CON, Control.
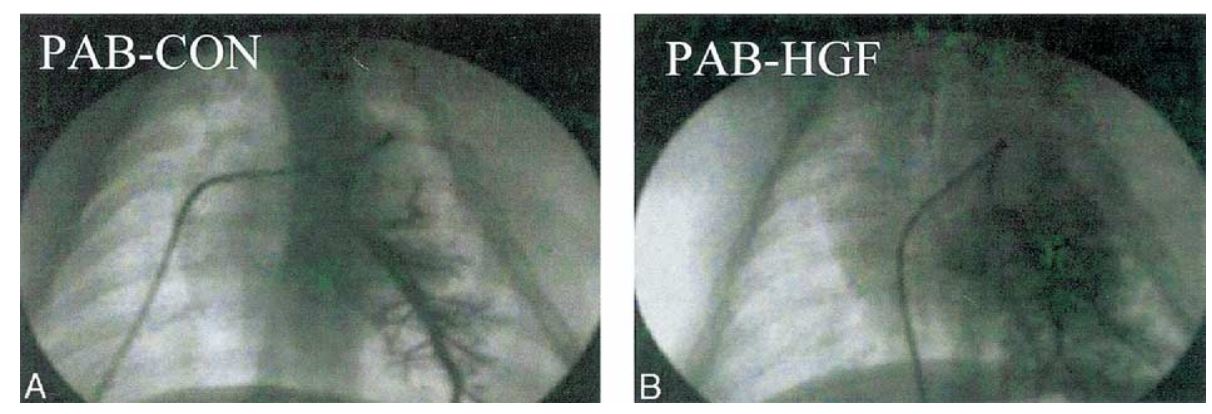

Figure 2. Angiographic changes in the pulmonary artery. Selective angiogram for the left pulmonary artery transfected with the HGF gene (B) or mock vector (A) 7 days after gene transfection.

various growth factors indicating angiogenesis can be applied as a treatment for ischemic coronary and peripheral artery disease, ${ }^{7,8}$ so-called "therapeutic angiogenesis."

Hepatocyte growth factor (HGF), which was originally purified and cloned as a potent mitogen for hepatocytes, ${ }^{9}$ acts as an organotrophic factor for the regeneration and protection of organs, including the liver, ${ }^{10}$ kidney, ${ }^{11}$ heart, ${ }^{12,13}$ and lung. ${ }^{14,15}$ Furthermore, HGF has an angiogenic effect on the ischemic heart ${ }^{16}$ and $\operatorname{limb}^{17}$ and the normal rat lung. ${ }^{18}$ On the basis of the above information, we hypothesized that in vivo gene transfection of the lung with HGF may have therapeutic effects for severe pulmonary vascular hypoplasia. In this study, we investigated the therapeutic potential of in vivo gene transfection with HGF using the piglet model of pulmonary vascular hypoplasia.

\section{Materials and Methods}

\section{Animal Care}

This study was carried out under the supervision of the Animal Research Committee in accordance with the Guidelines on Animal Experiments of Osaka University Medical School and the Japanese Government Animal Protection and Management Law.
Construction of Plasmid Bearing the Human HGF Gene To prepare an HGF expression vector, the cDNA for human HGF was inserted into the Not I site of the pUC-SR $\alpha$ expression vector plasmid. ${ }^{19}$ In this plasmid, expression of the HGF cDNA is regulated under the control of the SR $\alpha$ promoter. ${ }^{20} \mathrm{We}$ also constructed a control expression vector lacking the HGF gene.

\section{Preparation of Hemagglutinating Virus of Japan Envelope Vector}

The preparation of the hemagglutinating virus of Japan (HVJ) envelope vector is described by Kaneda and associates. ${ }^{21}$ In brief, stored virus was suspended in $30 \mu \mathrm{L}$ of TE solution $(10 \mathrm{mmol} / \mathrm{L}$ Tris-HCl, pH 8.0, $1 \mathrm{mmol} / \mathrm{L}$ EDTA). The virus suspension was mixed with $200 \mu \mathrm{g}$ of plasmid DNA and $5 \mu \mathrm{L}$ of detergent. The mixture was spun at $18,500 \mathrm{~g}$ for 15 minutes at $4^{\circ} \mathrm{C}$. After the pellet had been washed with $1 \mathrm{~mL}$ balanced salt solution to remove the detergent and unincorporated DNA, the envelope vector was suspended in $1 \mathrm{~mL}$ of phosphate-buffered saline (PBS) solution and used for subsequent experiments.

Surgical Approach and Left Pulmonary Artery Banding Female piglets weighing $5 \mathrm{~kg}$ were anesthetized by intraperitoneal injection of $50 \mathrm{mg} / \mathrm{kg}$ ketamine (Sankyo Co, Ltd, Tokyo, Japan), 5 
$\mathrm{mg} / \mathrm{kg}$ xylazine (Bayer AG, Leverkusen, Germany), and $5 \mathrm{mg} / \mathrm{kg}$ propionylpromazine (Bayer). The piglets were intubated and ventilated with a ventilator (MERA MD-705XL, Medical Instrument Mfg Co, Ltd, Tokyo, Japan). Breaths were administered at a rate of 30 per minute with a tidal volume of $130 \mathrm{~mL}$ with $40 \%$ oxygen and 2\% Sevofrane (Maruishi Pharmaceutical. Co, Ltd, Osaka, Japan). The piglets then underwent left thoracotomy. After the azygos vein was divided, the left pulmonary artery was dissected out and banded with polyester tape (circumference: $8 \mathrm{~mm}$ ). After hemostasis, the thoracotomy was closed in 3 layers. The piglets were extubated and allowed to recover in a warm, oxygenated environment.

\section{Gene Transfection of the Left Lung via the Pulmonary Artery}

Seven days after pulmonary artery banding, the piglets were anesthetized again and underwent lower partial median thoracotomy. A balloon catheter (CI-213; Harmac Medical Products, Inc, Buffalo, $\mathrm{NY}$ ) was inserted from the right ventricle into the left main pulmonary artery, where it was secured, and then selective left pulmonary artery angiography was performed. Next, the HVJ envelope-plasmid complex (20 mL, containing $2 \mathrm{mg}$ of cDNA) was infused via the catheter, after the occlusion of the left pulmonary artery flow by balloon inflation for 3 minutes. The catheter was then removed, the sternotomy was closed, and the soft tissues and skin were closed in layers. The animal was allowed to recover in a warm, oxygenated environment. The expression vector bearing the human HGF cDNA was transfected into 12 piglets (H group) and the vector without HGF was transfected into another 12 piglets, which served as the controls $(\mathrm{C}$ group). In addition, 4 piglets, which received no treatment, served as normal controls.

\section{Pulmonary Angiography and Pulmonary Artery \\ Occlusion Test}

To assess the change in pulmonary vasculature and pulmonary resistance in the left lung after gene transfection, we performed a pulmonary angiography and a pulmonary artery clamp test that was designed on the basis of our clinical experience. ${ }^{22}$ In brief, piglets were anesthetized, intubated, and ventilated again. A midline thoracotomy was carefully performed. A balloon catheter was inserted from the right ventricle and secured in the left main pulmonary artery. Left pulmonary angiography was then performed. The same catheter was then secured in the right main pulmonary artery, and the balloon was inflated, which occluded the right pulmonary artery. The right ventricular systolic pressure before and after occlusion was measured by puncture with a 22-gauge needle connected to a transducer (Terumo Medical Corporation, Somerset, NJ) and polygraph system (Nihon Kohden Co, Tokyo, Japan). This measurement was performed 3 times, and the increase in right ventricular pressure $(\triangle \mathrm{RVP})$ was calculated. The heart and lungs were then resected en bloc, and the lungs were cleared of blood by infusing cold PBS solution through a catheter positioned in the main pulmonary artery. For enzyme-linked immunoassay, blocks of lung tissue were obtained from all samples in each group. Then, all tissue samples subjected to histologic analysis were fixed in ethanol instilled at $20 \mathrm{~mm} \mathrm{Hg}$ of pressure thorough the main stem bronchus and kept for 24 hours on a constant pressure system. Four piglets in each group were evalu- ated 4, 7, and 14 days after the transfection. The surgeon performing the procedure was blinded to the treatment status (ie, HGF or control) of each animal until the end of the study.

\section{Immunohistochemical Analysis}

Tissue specimens obtained from the hilum of the left lung were fixed in ethanol, embedded in paraffin, and sectioned. The sections were immunohistochemically stained with a rabbit polyclonal antibody against factor VIII, which is a specific marker of endothelial cells. The number of factor VIII-positive arteries that were more than $100 \mu \mathrm{m}$ in diameter was counted under a microscope for 10 randomly selected fields per specimen. The arterial density was determined as the average number of factor VIII-positive arteries along 1 terminal bronchiole per $1 \mathrm{~mm}^{2}$. The pathologist reviewing the sections was blinded to the experimental group until the end of the analysis.

\section{Assay of Human HGF}

To perform the enzyme-linked immunosorbent assay (ELISA) of human HGF in the treated lung, blocks of the lung from each group were obtained 4,7 , and 14 days after gene transfection. The level of human HGF in the lung was estimated with an anti-human HGF monoclonal antibody (Institute of Immunology, Tokyo, Japan). The antibody that we used in the ELISA specifically detects human HGF but not endogenous piglet HGF.

\section{Statistical Analysis}

All values are expressed as the mean \pm SEM. The statistical differences in data obtained by pressure assessment after pulmonary artery banding and ELISA were determined by the Student $t$ test. Statistical differences in the other data were assessed by 1-way analysis of variance (ANOVA) followed by the Bonferroni post hoc test.

\section{Results}

\section{Evaluation of Pulmonary Vascular Hypoplasia After Pulmonary Artery Banding}

Seven days after pulmonary artery banding, selective left pulmonary arteriography demonstrated marked decrease in pulmonary vasculature after pulmonary artery banding compared with the normal control (Figure 1, A). Diminishing peripheral pulmonary arteries and dilation of left main pulmonary artery were featured in this model. Right pulmonary artery occlusion test, in which the left lung received total blood flow from the right ventricle, also revealed a significant increase of right ventricular systolic pressure 7 days after the procedure compared with the normal control (Figure $1, B)$.

\section{Evaluation of HGF Gene Transfection}

Next, we evaluated the effect of HGF gene transfer in this model. An enzyme immunoassay demonstrated a human HGF protein expression in all of the HGF-transfected lungs 4 and 7 days after transfection $(0.35 \pm 0.02,0.37 \pm 0.02$ $\mathrm{ng} / \mathrm{g}$ tissue, respectively), whereas it was not detected in the control lung $(P<.01$ vs control; Student $t$ test). Human 


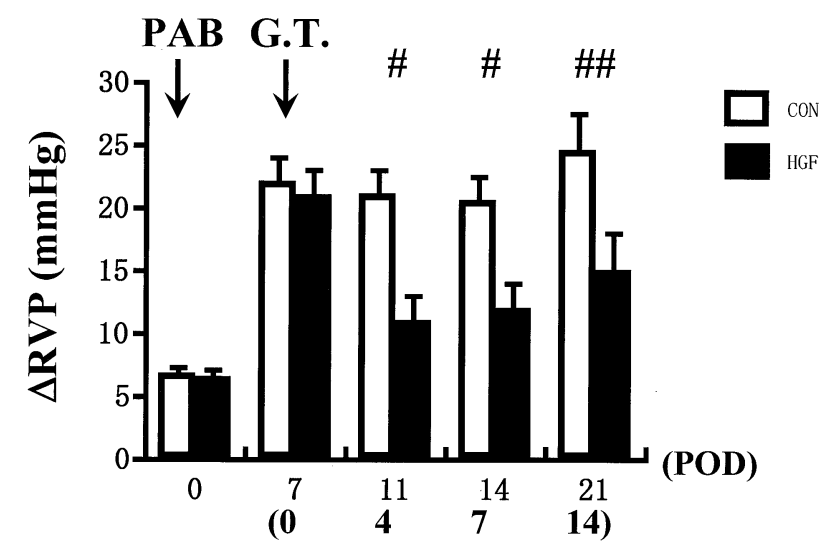

Figure 3. Changes in the pulmonary vascular resistance of the left lung by a right pulmonary artery occlusion test. The increase in right ventricular pressure $(\Delta R V P)$ was attenuated in $\mathrm{H}$ group on days 4,7 , and 14 after gene transfection (G.T.). Each value represents the mean \pm SEM of values obtained using 4 piglets at each time point in each group. \#\# $<.01, \# P<.05$ versus control (CON) (ANOVA). The black bar indicates HGF gene-transfected lung ( $=4)$; the white bar indicates control lung $(n=4)$.

HGF could not be detected on days 14 in either HGFtransfected lungs or control lungs.

Selective left pulmonary arteriogram demonstrated progressive pulmonary vascular hypoplasia of the left lung in the control group (Figure 2, A, Video 1) 7 days after gene transfection, whereas in the piglets that received HGF gene therapy, left pulmonary angiogram demonstrated marked increase in pulmonary vasculature of the left lung (Figure 2, $B$, Video 1). These angiographic differences were observed at 4 and 14 days after gene transfection.

A right pulmonary artery occlusion test revealed that the $\Delta$ RVP had not changed after gene transfection in the $\mathrm{C}$ group but had significant attenuation in the $\mathrm{H}$ group. This significant difference continued for 14 days after gene transfection (Figure 3).

Histologic examination revealed that there was an increase of the number of pulmonary arteries (Figure $4, B$ ) compared with the control group (Figure 4, A). Furthermore, a marked increase of factor VIII-positive pulmonary arteries along a single terminal bronchiole was observed in the $\mathrm{H}$ group (Figure 4,C) on days 4, 7, and 14 after gene transfection.

\section{Discussion}

In the present study, we demonstrated that in vivo transfection of the lung with the HGF gene increased pulmonary vasculature in a piglet model of pulmonary vascular hypoplasia. This conclusion is supported by the following evidence: (1) pulmonary arteriography showed the increase of pulmonary vasculature; (2) the number of factor VIII-positive pulmonary arteries significantly increased; (3) left pul- monary vascular resistance, assessed by the pulmonary artery occlusion test, decreased after HGF gene transfection.

We evaluated pulmonary arteries in the left lung after transfection of HGF gene, both angiographically and histologically. There was no angiographic or histologic vascular abnormality, such as pulmonary arteriovenous fistula, and no significant decrease in oxygen saturation in the arterial blood after HGF gene transfection (data not shown). We could not see any general side effects during or after gene transfection of HGF. The pulmonary arteries induced by HGF gene transfection in this piglet model of pulmonary vascular hypoplasia seemed to be a relatively large size compared with that reported in coronary $^{12}$ or peripheral ${ }^{17}$ artery disease. Our angiographic data showed an increase in the number of pulmonary arteries, and histologic analysis revealed an increase in the number of pulmonary arteries in the transfected lung. Further investigation is needed to confirm the mechanisms of this vascular development.

As to the model of pulmonary vascular hypoplasia, there is no established animal model for creating pulmonary vascular hypoplasia. As Lambert and colleagues ${ }^{23}$ reported the usefulness of left pulmonary artery banding for creating pulmonary vascular hypoplasia, we performed left pulmonary artery banding in neonatal piglets. In this model, angiographic and histologic analysis demonstrated significant vascular change 7 days after banding and showed progressive pulmonary vascular hypoplasia. Then, we performed HGF gene transfection 7 days postoperatively. Our histologic data showed pulmonary angiogenesis consistent with the result of Lambert and associates, ${ }^{23}$ and angiographic data demonstrated physiologic increase of pulmonary vasculature even in the presence of left pulmonary artery banding. This suggests that a reduced but persisting blood supply is sufficient for peripheral pulmonary vascular development in the presence of overexpressed HGF. HGF gene transfection decreased the pulmonary vascular resistance when blood flow increased, even in the presence of left pulmonary artery occlusion. About this mechanism, we speculate that when pulmonary blood flow is increased, pulmonary vascular resistance largely depends not on the central pulmonary artery size but on the compliance of peripheral pulmonary vasculature, based on our clinical experience ${ }^{22}$ and another report. ${ }^{24}$ To compare the effect of HGF gene transfection with that of debanding or placement of a shunt to the left pulmonary artery is a great concern. Further investigation is mandatory to evaluate how this strategy contributes to the clinical aspects for the treatment of severe pulmonary vascular hypoplasia.

The main limitation of this study is that our data were obtained from piglets that had received pulmonary artery banding after birth. There are some differences in the condition of the pulmonary vasculature between this model and lungs that are clinically diseased from the fetal period, such as in the tissue levels of growth factors and endothelial function. Further investigation under conditions that repre- 


\section{PAB-CON}

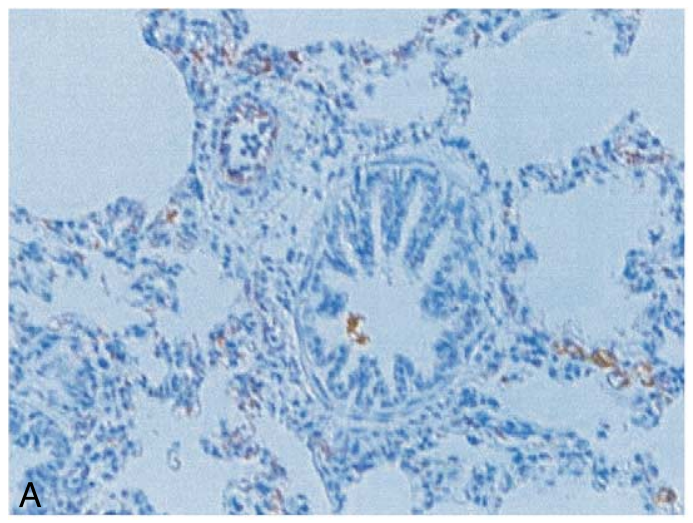

\section{PAB-HGF}
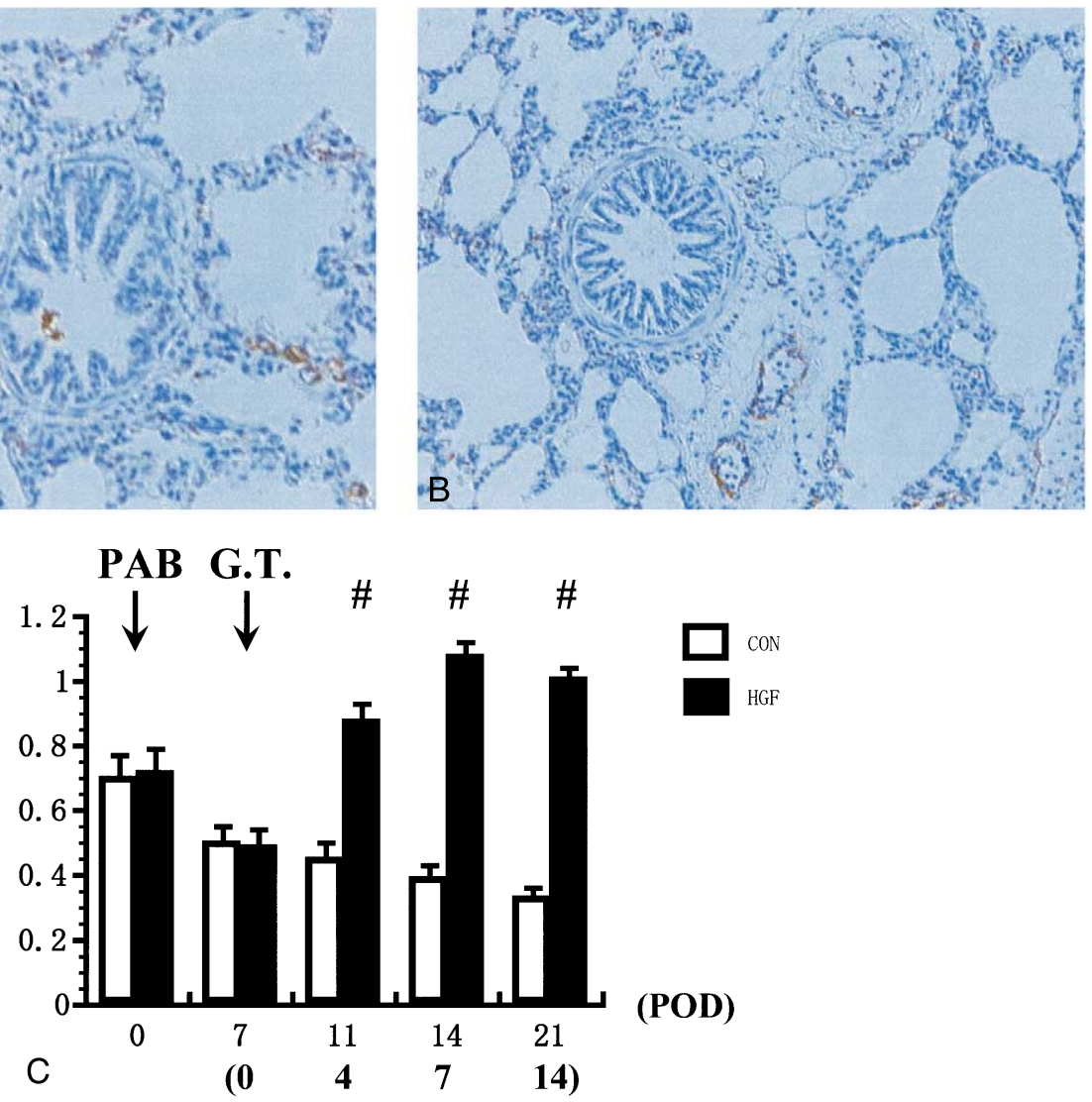

Figure 4. Changes in the number of arteries in the left lung. $A$ and $B$, Pulmonary arteries along terminal bronchiole in the left lung transfected with HGF gene $(B)$ or mock vector $(A)$ at 7 days after gene transfection. (Magnifications $A, B: \times 100$.) C, Number of pulmonary arteries along the terminal bronchial. Each value represents the mean \pm SEM of values obtained using 4 piglets in each group. \#P<.01 versus control (CON). The black bar indicates HGF gene-transfected lung ( $=4)$; the white bar indicates control lung $(n=4)$.

sent the damaged or underdeveloped lung, including pulmonary banding in the gestational period, will need to be carried out. However, this model may be relevant to the same situation of pulmonary vascular disease clinically evaluated.

Used in combination with a conventional surgical procedure such as systemic-pulmonary shunt and unifocalization of major aortopulmonary collateral arteries, this strategy may increase pulmonary blood flow and decrease the pulmonary vascular resistance. Synergistic effects of these strategies should be expected and also investigated using a combination therapy of shunt and gene transfection. Although further investigation is required to confirm the therapeutic potential of this strategy, our results showed the possibility for treating patients with severe pulmonary vascular hypoplasia.

In summary, in vivo gene transfection of the lung with HGF by means of pulmonary arterial injection of the HVJ envelope vector increased pulmonary vasculature angiographically, ameliorated the increase in right ventricular pressure when the right pulmonary artery was occluded, and increased the histologic number of pulmonary arteries, suggesting the development of left pulmonary vasculature. These data suggest that gene transfection with HGF may become a new therapy for patients with severely hypoplastic pulmonary vasculature.

We thank Mrs Masako Yokoyama and Mr Shigeru Matsumi for technical assistance.

\section{References}

1. Blalock A, Taussig HB. The surgical treatment of malformation of the heart in which there is pulmonary stenosis or pulmonary atresia. JAMA. 1945;128:189-202.

2. Stamm C, Friehs I, Duebemer LF, Zurakowski D, Mayer JE Jr, Jonas RA, et al. Improved results of the modified Fontan operation in patients with heterotaxy syndrome. Ann Thorac Surg. 2002;74:1967-77. 
3. Azakie A, Merklinger SL, Williams WG, VanArsdell GS, Coles JG, Adatia I. Improving outcomes of the Fontan operation in children with atrial isomerism and heterotaxy syndromes. Ann Thorac Surg. 2001; 72:1636-40.

4. Duncan BW, Mee RB, Prieto LR, Rosenthal GL, Mesia CI, Qureshi A, et al. Staged repair of tetralogy of Fallot with pulmonary atresia and major aortopulmonary collateral arteries. J Thorac Cardiovasc Surg. 2003;26:694-702.

5. Yagihara T, Yamamoto F, Nishigaki K, Matsuki O, Uemura H, Ishizaka $\mathrm{T}$, et al. Unifocalization for pulmonary atresia with ventricular septal defect and major aortopulmonary collateral arteries. J Thorac Cardiovasc Surg. 1996;112:392-402.

6. Hashmi A, Abu-Sulaiman R, McCrindle BW, Smallhorn JF, Williams WG, Freedom KM. Management and outcomes of right atrial isomerism: a 26-year experience. J Am Coll Cardiol. 1998;31:1120-6.

7. Losordo DW, Vale PR, Symes JF, Dunnington CH, Esakof DD, Maysky $\mathrm{H}$, et al. Gene therapy for myocardial angiogenesis: initial clinical results with direct myocardial injection of phVEGF165 as sole therapy for myocardial ischemia. Circulation. 1998;98:2800-4.

8. Isner JM, Pieczek A, Schainfeld R, Blair R, Haley L, Asahara T, et al. Clinical evidence of angiogenesis after arterial gene transfer of phVEGF in patient with ischemic limb. Lancet. 1996;348:370-4.

9. Nakamura T, Nishizawa T, Hagiya M, Seki T, Shimonishi M, Sugimura A, et al. Molecular cloning and expression of human hepatocyte growth factor. Nature. 1989;342:440-3.

10. Michalopoulos GK, DeFrances MC. Liver regeneration. Science. 1997;276:60-6.

11. Mizuno S, Kurosawa T, Matsumoto K, Mizuno-Horikawa Y, Okamoto M, Nakamura T. Hepatocyte growth factor prevents renal fibrosis and dysfunction in a mouse model of chronic renal disease. J Clin Invest. 1998;101:1827-34.

12. Funatsu T, Sawa Y, Ohtake S, Takahashi T, Matsumiya G, Matsuura $\mathrm{N}$, et al. Therapeutic angiogenesis in the ischemic canine heart induced by myocardial injection of naked complementary DNA plasmid encoding hepatocyte growth factor. J Thorac Cardiovasc Surg. 2002; 124:1099-105.

13. Nakamura T, Mizuno S, Matsumoto K, Sawa Y, Matsuda H, Nakamura T. Myocardial protection from ischemia/reperfusion injury by endogenous and exogenous HGF. $J$ Clin Invest. 2000;106:1511-9.

14. Ohmichi H, Matsumoto K, Nakamura T. In vivo mitogenic action of HGF on lung epithelial cells: pulmotrophic role in lung regeneration. Am J Physiol. 1996;270:L1031-9.
15. Sakamaki Y, Matsumoto K, Mizuno S, Miyoshi S, Matsuda H, Nakamura $\mathrm{T}$. Hepatocyte growth factor stimulates proliferation of respiratory epithelial cells during postpneumectomy compensatory lung growth in mice. Am J Respir Cell Mol Biol. 2002;26:525-33.

16. Aoki M, Morishita R, Taniyama Y, Kida I, Moriguchi A, Matsumoto $\mathrm{K}$, et al. Angiogenesis induced by hepatocyte growth factor in noninfarcted myocardium and infarcted myocardium: up-regulation of essential transcription factor for angiogenesis, ets. Gene Ther. 2000; 7:417-27.

17. Taniyama Y, Morishita R, Aoki M, Nakagami H, Yamamoto K, Yamazaki K, et al. Therapeutic angiogenesis induced by human hepatocyte growth factor gene in rat and rabbit hindlimb ischemia models: preclinical study for treatment of peripheral arterial disease. Gene Ther. 2001;8:181-9.

18. Ono M, Sawa Y, Matsumoto K, Nakamura T, Kaneda Y, Matsuda H. In vivo gene transfection with hepatocyte growth factor via the pulmonary artery induces angiogenesis in the rat lung. Circulation. 2002; 106(Suppl I):I264-9.

19. Seki T, Hagiya M, Shimonishi M, Nakamura T, Shimizu S. Organization of human hepatocyte growth factor-encoding gene. Gene. 1991; 102:213-9.

20. Kaneda Y, Iwai K, Uchida T. Increased expression of DNA cointroduced with nuclear protein in adult rat liver. Science. 1989;243: 375-8.

21. Kaneda Y, Nakajima T, Nishikawa T, Yamamoto S, Ikegami H, Suzuki N, et al. Hemagglutinating virus of Japan (HVJ) envelope vector as a versatile gene delivery system. Mol Ther. 2002;6:219-26.

22. Ono M, Kishimoto H, Kawata H, Miura T, Funatsu T, Iwai S, et al. Usefulness of pulmonary artery clamp test for the evaluation of pulmonary vascular resistance in isomerism heart with pulmonary venous obstruction. Pediatr Cardiol Card Surg. 2001;17:709-14.

23. Lambert V, Michel R, Mazmanian GM, Dulmet EM, Capderon A, Herve $\mathrm{P}$, et al. Induction of pulmonary angiogenesis by adenoviralmediated gene transfer of vascular endothelial growth factor. Ann Thorac Surg. 2004;77:458-63.

24. Sawatari K, Imai Y, Kurosawa H, Fukuchi S, Kawada M, Matsuo $\mathrm{K}$, et al. New selection criterion for Fontan procedure: pulmonary artery clamping test and pulmonary vascular resistance in increased pulmonary blood flow. Nippon Kyobu Geka Gakkai Zasshi. 1989; 7:208-17. 